\title{
Ciencia pura versus ciencia aplicada: la fuerza de la tradición positivista en la ciencia brasileña a comienzos del siglo $\mathrm{Xx}^{*}$
}

Luiz Otávio Ferreira

UNIVERSTDAD DEL ESTADO DE RÍO DE JANEIRO (BRASIL)

\begin{abstract}
A finales del siglo XIX, el positivismo fue una referencia obligatoria para los intelectuales y científicos brasileños, entre los que se propagó la función de la ciencia aplicada y sirvió como instrumento de crítica al orden social vigente basada en una ideología que consideraba necesaria la modernización para el desarrollo científico del país.
\end{abstract}

$\mathrm{E}$ I debate en torno a las proposiciones científicas del positivismo comtiano movilizó, durante las dos primeras décadas del siglo $\mathrm{XX}$, a ingenieros, matemáticos, astrónomos y profesores de ciencias físicas y naturales brasileños vinculados con la antigua Escuela Politécnica de Río de Janeiro. En el presente trabajo se procura reconstituir la polémica científica entre los politécnicos, poniendo especial atención en la cuestión de ciencia pura frente a ciencia aplicada, en el contexto del movimiento intelectual

* Traducción de Martín Mur U. que dio origen a la Academia Brasileña de las Ciencias en 1922.

Erno Paulinyi ${ }^{1}$ trató de definir las principales características del movimiento intelectual que auspició la fundación, en 1916, de la Sociedad Brasileña de las Ciencias, entidad que más tarde, en 1922, se transformaría en la actual Academia Brasileña de las Ciencias. En opinión de este autor, el movimiento intelectual que dio origen a la Academia Brasileña de las Ciencias podría caracterizarse por la crítica de los hábitos intelectuales característi-

\footnotetext{
1 Paulinyi, Esboço, 1981, pp. 11-12.
} 
cos de una formación jurídica y literaria (el bachillerismo), así como por el deseo de dinamizar la investigación científica en Brasil a través de una cruzada antipositivista, toda vez que la filosofía de Augusto Comte, ampliamente difundida entre los hombres de ciencia brasileños, ya se consideraba obsoleta. Solamente con la superación simultánea del bacbillerismo y del positivismo comtiano quedaría garantizada la posibilidad de florecimiento del interés por la investigación científica pura entre los brasileños.

Al contrario de lo acaecido en el Instituto Politécnico Brasileño (1862), entidad que se caracterizó por la defensa de los intereses profesionales de los ingenieros a partir de la idea de que la modernización de la sociedad brasileńa iba a depender de la acción de los hombres científicamente preparados, ${ }^{2}$ los científicos politécnicos que en 1916 fundaron la Sociedad Brasileña de las Ciencias rechazaban categóricamente la idea de la utilidad práctica de la ciencia. Éstos dejarían establecido que la finalidad principal de aquella sociedad científica sería la de contribuir al desarrollo de las ciencias $y$ de sus aplicaciones que no tuvieran un carácter profesional. ${ }^{3}$ En 1917 fue reafirmada la exclusión de la ciencia aplicada de los objetivos institucionales de la asociación. Desde entonces quedó definido que el objetivo de la Sociedad Brasileña de las Ciencias sería el de contribuir al desarrollo de las ciencias y de sus aplicaciones que no tuvieran un carácter in-

\footnotetext{
${ }^{2}$ Ferreira, "Politécnicos", 1989.
}

${ }^{3}$ Paulinyi, Esboço, 1981, p. 13. dustrial o comercial, ${ }^{4}$ orientación que fue mantenida hasta 1930.

¿Cuáles tendrían que haber sido los motivos que llevaran a los fundadores de la Academia Brasileña de las Ciencias a la defensa de una forma tan radical de idealismo científico? Irno Paulinyi sustenta la hipótesis de que este idealismo científico fue esencialmente una reacción de los sectores más esclarecidos de la comunidad científica brasileña contra el obstáculo más poderoso al desarrollo de la ciencia nacional a principios del siglo $\mathrm{xx}$ : el positivismo comtiano.

No obstante, es posible una interpretación diferente. Podemos decir que la referencia constante al positivismo -tanto para criticarlo como para defenderlo-, fue una actitud típica e históricamente fechada de los científcos e intelectuales brasileños de principios del siglo Xx. Para este caso resulta perfectamente aplicable el postulado de Pierre Bourdieu según el cual "sería posible determinar áreas y generaciones de intelectuales, a través del conjunto de cuestiones obligatorias que definen el campo cultural de una determinada época". 5 El tema dcl positivismo fue una de las cuestiones obligatorias en torno a la cual se organizaba el campo científico brasileño.

De ese modo, las críticas enderezadas contra el positivismo comtiano, formuladas por el grupo de los politécnicos fundadores de la Academia Brasileña de las Ciencias, deben ser entendidas como una estrategia de construcción de la identidad de un

${ }^{4}$ Ibid.

${ }^{3}$ Bourdieu, Economia, 1987, p. 206. 
nuevo tipo intelectual: el científico puro. La afirmación de la identidad social científico puro dedicado a la investigación básica se obtendría mediante la negación de los valores y hábitos tradicionales reinantes en los medios científicos e intelectuales brasileños donde predominaba una visión instrumental de la ciencia.

LA ENSEÑANZA DE IA INGENIERIA Y LA TRADICIÓN POSITIVISTA

A partir de las postrimerías del último cuarto del siglo XIX -y adentrándonos en el XX-, Brasil presenció una serie de iniciativas en el ámbito científico que implicaron tanto la creación de nuevos espacios institucionales como la reformulación de los previamente existentes. Son ejemplos del primer caso la Comisión Geológica de Brasil (1875), la Escuela de Minas de Ouro Preto (1875), la Comisión Geológica de São Paulo (1886), la Estación Agronómica Imperial de Campinas (1887), el Museo Paraense (1871), el Laboratorio de Fisiología Experimental del Museo Nacional (1878), la Policlínica de Río de Janeiro (1882), el Instituto Pasteur de Río de Janeiro (1888), la Sociedad de Medicina y Cirugía de Río de Janeiro (1886), los tres primeros Congresos Médicos Nacionales (1888, 1889 y 1890), el Instituto Bacteriológico de São Paulo (1892), la Escuela Politécnica de São Paulo (1893), el Museo Paulista (1894), el Instituto Soroterapéutico de Manguinhos (1899), el Instituto Butantã (1901), el Servicio Geológico y Mineralógico de Brasil (1907), entre otros. Del segundo caso, citemos como ejemplo el desmembramiento del Observatorio Imperial de la Escuela Central de Ingeniería (1871), la transformación de la Escuela Central de Ingeniería en la Escuela Politécnica de Río de Janeiro (1874), la revitalización del Colegio Pedro II (1876 y 1878), las reformas al plan de estudios de las Facultades de Medicina de Río de Janeiro y de Salvador (1879 y 1884).

Fue en asociación con el proceso de institucionalización de la ciencia de naturaleza predominantemente aplicada, como el positivismo se propagó entre los científicos e intelectuales brasileños, sirviendo al mismo tiempo como instrumento de crítica del orden social vigente, crítica basada en el régimen monárquico y en la esclavitud, así como en la ideología cientificista que difundía la idea de que la modernización social estaba directamente relacionada con el desarrollo científico del país.

Para entender la historia del positivismo brasileño resulta oportuno recordar que el movimiento positivista en su país natal, esto es, en Francia, desde la muerte de Augusto Comte en 1857, se dividió en dos facciones: una ortodoxa, encabezada por Pierre Lafitte, sucesor de Comte en la función de gran sacerdote de la Religión de las Humanidades, y otra heterodoxa, cuyo principal exponente fue el filólogo Êmile Littré. Las divergencias entre positivistas ortodoxos y heterodoxos son el resultado de la propia dicotomía de la obra comtiana, dividida entre la valorización de la razón científica y la prédica de una nueva religión. Para los positivistas ortodoxos, la misión del positivismo era el 
fomento de una profunda reforma moral de la sociedad, por vía de la implantación de la Religión de las Humanidades. Y en seguida, para los positivistas heterodoxos lo fundamental era la instauración definitiva del positivismo científico en las diferentes áreas del conocimiento humano.

En Brasil se reprodujo la división clásica del positivismo. En 1874 se fundó la Sociedad Positivista de Río de Janeiro, entidad que reunía a profesionales liberales de formación científica -ingenieros, médicos, matemáticos, profesores en general- bajo una orientación heterodoxa. En 1881, con el ascenso de Miguel Lemos y 'Teixeira Mendes al mando de la entidad, tal orientación heterodoxa fue abandonada y la Sociedad Positivista de Río de Janeiro se vio transformada en el Apostolado Positivista del Brasil, representante oficial de la Religión de las Humanidades. La opción ortodoxa que se impuso a la sociedad hizo que varios militantes positivistas heterodoxos abandonaran la asociación. A partir de entonces, podemos decir que el positivismo brasileño se caracterizó por la existencia de un pequeño, cohesionado y activo grupo positivista ortodoxo y por un sinnúmero de positivistas heterodoxos cuya militancia intelectual y política fue expresiva, aunque no formalmente organizada.

La historia de la construcción de una tradición positivista en Brasil, está íntimamente relacionada con la enseñanza de la ingeniería durante el Imperio. Por más que las otras instituciones de enseñanza superior existentes en el país -las facultades de medicina localizadas en Río de Ja-

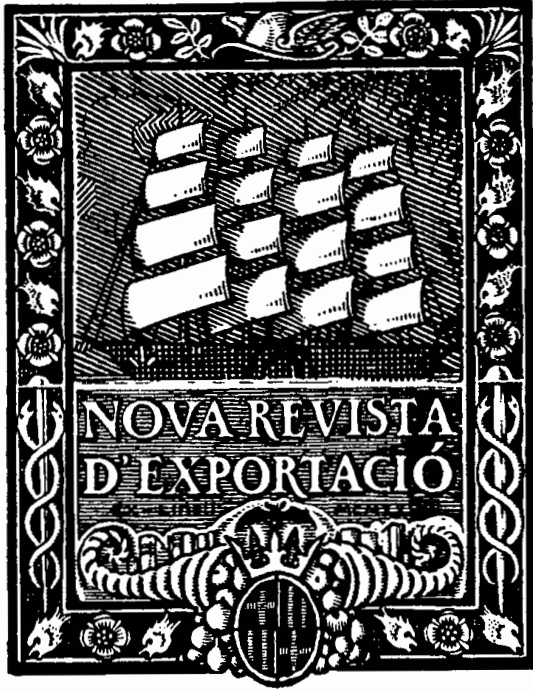

neiro y en Salvador, y las facultades de derecho ubicadas en São Paulo y Recife- también habían servido como focos de irradiación del positivismo, las escuelas de ingeniería fueron, de hecho, el ambiente en el que el positivismo científico fue institucionalizado. Los profesores de estas escuelas se aferraron sobre todo a la conceptualización y el rigor de sistematización que encontramos en las obras filosóficas y matemáticas de Augusto Comte. Gracias a la acción de estos profesores, el positivismo, a la postre, acabó influyendo en la enseñanza de las cien. cias físicas y matemáticas, en Brasil, hasta fines del siglo XIX, y convirtién. dose en una barrera para el desarrollo de trabajos originales en estas áreas. ${ }^{6}$

\footnotetext{
${ }^{6}$ Dantes, "Fases", 1988, p. 269.
} 


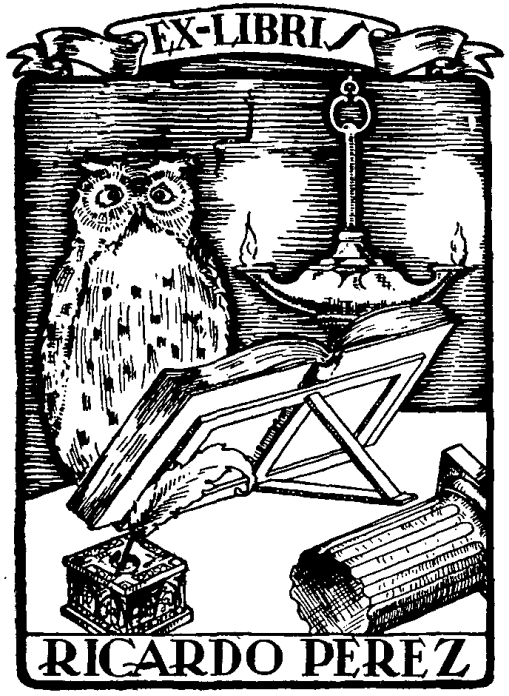

En 1873 se creó la Escuela Politécnica de Río de Janeiro, institución que venía a sustituir a la antigua Escuela Central de Ingeniería que venía funcionando desde 1858 . Un año después, el Ministerio del Imperio, en cuya cartera durante el régimen imperial estaban comprendidos los asuntos relativos a la educación, estableció los estatutos de la nueva escuela de ingeniería, en los que se determinaba la siguiente estructura de cursos: ciencias físicas y naturales, ciencias físicas y matemáticas, ingeniería geográfica, ingeniería civil, ingeniería de minas, y artes y manufacturas. Con el proyecto original, la Escuela Politécnica tenía por finalidad formar, además de ingenieros en diversas especialidades, bachilleres y "doctores" en ciencias físicas y naturales. No obstante, los cursos científicos gozaron de una corta vida; en 1896, ya bajo el régimen republicano, fueron abolidos. A partir de entonces la Escuela Politécnica de Río de Janciro pasó a dedicarse exclusivamente a la formación de ingenieros en las siguientes especialidades: ingeniería civil, ingeniería de minas, ingeniería industrial, ingeniería mecánica $c$ ingeniería agronómica.

Ia decisión de eliminar los cursos científicos de la Escuela Politécnica parece haber sido tomada con base en la reducida aceptación de tales cursos. Entre 1874 y 1896, la distribución de los estudiantes fue la siguiente:

Cursos

Nímero de egresados

Bachilleres en ciencias físicas y matemáticas 32

Bachilleres en ciencias físicas y naturales

Ingenieros industriales (artes

y manufacturas)

Ingenieros de minas

13

Ingenieros civiles

08

705

Ingenieros geógrafos

209

Total

1004

¿Qué relación podía haber entre la eliminación de los cursos científicos y la crítica al positivismo iniciada en 1896 dentro de los muros de la escuela del Largo do São Francisco? Para algunos profesores y alumnos, la eliminación de esos cursos era el resultado de la vigencia, entre los politécnicos, de una visión pragmática de la cicncia impuesta por el positivismo. Aun cuando podamos explicar la preferencia ma- 
yoritaria de los alumnos por la ingeniería civil, ${ }^{7}$ relacionando este hecho con la cuestión de la reforma urbana y sanitaria de Río de Janeiro, no deja de ser verdad que la orientación pedagógica oficial basada en las enseñanzas de Augusto Comte fue un grave obstáculo para el desarrollo de cursos eminentemente científicos. La enseñanza de las ciencias, sobre todo de las matemáticas, fue acentuadamente positivista, y eso tuvo implicaciones institucionales que quedaron registradas en la memoria de quienes asistieron a la Escuela Politécnica durante ese periodo. Roberto Marinho de Azevedo, matemático que asistió a la Escuela Politécnica a principios del siglo $\mathrm{XX}$ y que formó parte de la generación de científicos críticos del positivismo, ofrece el siguiente relato de la situación:

Aferrados todavía al pasado, discutíamos las concepciones de Leibniz, de Newton y de Lagrange sobre el cálculo infinitesimal. Nos interesábamos por los origenes. Guardábamos un intenso recuerdo de los grandes espíritus de los tiempos pasados: Kepler, Galileo, Newton, Leibniz, Laplace, Lagrange, d'Alembert. La influencia de Comte se hacía sentir en el curso de cálculo diferencial e integral de Ferreira Praga, así como en el de Licínio Cardoso, a la vez que en cursos particulares de matemáticas como los del Sr. Nazareth y del Sr. Guedes. La geometría analítica de Comte,

\footnotetext{
${ }^{7}$ En Brasil, desde la creación de la Escuela Central de Ingeniería en 1858, se instituyó una distinción entre el curso de ingeniería al que asistían los militares, $y$ el curso de ingeniería que era frecuentado por otros alumnos, y que pasó a ser designado como ingeniería civil.
}

esto es, la geometría general, como él prefería llamarla, era muy leída. ${ }^{\circ}$

Otro testimonio que nos da la proporción de la fuerza del positivismo en la enseñanza politécnica, fue el que nos dejó Leilo Gama, otro antipositivista que también asistió al curso de ingeniería a comienzos del siglo $\mathrm{XX}$ :

Para adaptarse a la orientación oficial, el estudiante tenía que abosorber prolijas obras didácticas, basadas, con la máxima fidelidad, en los esquemas de Augusto Comte. Justo es dejar constancia de que, dentro de los cuerpos docentes de la época, valiosas inteligencias, que dieron brillo a cátedras militares y civiles, bien pudieron haber modificado los rumbos de la enscñanza superior de las matemáticas. limpero, se dejaron poseer, digámoslo así, por el fanatismo intelectual que inspiraba el seductor filósofo francés. Este estado de ánimo, cristalizado en el sistema positivista, impedía la apertura de nuevos horizontes, de nuevas senclas que, entre tanto, comenzaban a despertar también la curiosidad de jóvenes estudiantes, de los candidatos a los cursos de ingeniería. ${ }^{9}$

Para confirmar la veracidad de las anteriores declaraciones basta con citar a Licínio Cardoso, catedrático de mecánica racional y el más destacado re-presentante del positivismo politécnico, que confirma esa adhesión incondicional al positivismo comtiano al esclarecer su interpretación respecto a la evolución de la ciencia matemática en Brasil. Para él, 265.

${ }^{8}$ Citado por Lins, História, 1967, pp. 264 -

${ }^{9}$ Citado por Silva, Análises, 1994, p. 28. 
La ciencia estaba ya del todo constituida cuando, gracias a la fundación de las primeras academias, comenzó a ser estudiada en nuestra patria. Ya Descartes y Leibniz habian formulado la legislación matemática definitiva para la creación de la geometría general, producto del genio inductivo del filósofo francés, así como para la creación del método y el cálculo infinitesimales, producto de la genial intuición clel filósofo germano. También Lagrange había ya producido la elaboración sintética que, a través de la generalización inductiva del principio de d'Alembert, iba a reducir la mecánica a una fórmula general. Esto permite afirmar, a priori, que por muy bien dirigido y por más proficiente que haya sido entre nosotros el cultivo de la ciencia, éste jamás habrá contribuido al desarrollo de una cienca cuya evolución se hallaba ya realizada. ${ }^{10}$

La incómoda conclusión a que llegaba Licínio Cardoso era que resultaría improbable que los matemáticos brasileños efectuaran cualquier aportación original al desarrollo de la ciencia, por el simple hecho de que la matemática ya había alcanzado, a finales del siglo XVII, su estadio teórico definitivo. Indudablemente, el razonamiento de Cardoso se basaba en las objeciones de Comte respecto a las nuevas teorías científicas que surgían en el siglo XIX. Comte se mostró hostil al cálculo de las probabilidades inventado por Laplace; en astronomía censuró todo esfuerzo por determinar la constitución física de los astros; en física no aprobó los intentos encaminados a determinar la constitución de

${ }^{10}$ Cardoso, Evoluçāo, 1887, p. 3. la materia; en biología condenó toda teoría de evolución de las especies. Todas estas líneas de investigación científica se hallaban en contradicción, según Comte, con el objetivo fundamental de la ciencia, el cual debe ser la demostración de las leyes o relaciones constantes entre los fenómenos, ignorando cualquier especulación sobre la naturaleza íntima y/o las causas primeras de los datos naturales.

\section{REACCIÓN FRENTE AL POSITIVISMO} EN la EscueIa Pol.tT́tenica

El artículo del joven ingeniero y matemático Otto de Alencar, "Algunos errores de matemáticas en la síntesis subjetiva de A. Comte", publicado en 1898 en la revista de la Escuela Politécnica, es un documento decisivo para la comprensión de las relaciones que existían entre el positivismo y la ciencia en el Brasil de fines del siglo XIX. Más que una "denuncia" de las limitaciones teóricas de la matemática comtiana, el artículo indica el surgimiento, entre los politécnicos brasileños, de una concepción que no valorizaba el uso instrumental de la ciencia.

No fue tarea sencilla demostrar cuáles eran los "errores de matemáticas" de Comte, puesto que ello significaba ir en contra de un concepto de ciencia plenamente aceptado entre los los politécnicos, a pesar de sus implicaciones contradictorias para el desarrollo de la investigación científica nacional. Como casi todos los politécnicos de su época, Otto de Alencar fue también un positivista, habiendo 
sido asiduo asistente a las reuniones de la Sociedad Positivista de Río de Janeiro antes de que esta institución se convirtiera a la ortodoxia positivista. Por tanto, estaba familiarizado y, durante algún tiempo, comprometido con las ideas científicas de Augusto Comte. Esto explicaría la cautela con la que Otto de Alencar revistió su crítica a Comte. Tras la demostración minuciosa de los errores que contenían las proposiciones comtianas sobre la "teoría general de la curvatura", y que se encuentran en la Síntesis subjetiva, concluía su artículo diciendo que "desafortunadamente, a pesar de la autoridad de Augusto Comte, una proposición matemática no se impone al espíritu como objeto de fe". ${ }^{11}$

Otto de Alencar se empeñó en ampliar la divulgación de sus críticas buscándose aliados en la comunidad científica internacional. Al principio tuvo la intención de enviar un artículo en el mismo tenor, al editor de la revista francesa Enseignement Mathématique. Sin embargo, al saber que el editor de tal publicación periódica mostraba simpatía por las ideas de Augusto Comte, el matemático portugués Francisco Gomes Teixeira le aconsejó que presentara su trabajo al Journal de Sciencias Mathemáticas, Physicas e Naturaes, que se editaba en Portugal. Esta revista periódica publicó, en 1901, el artículo titulado "Quelques erreurs de Comte", en el cual Otto de Alencar profundizaba sus críticas a la matemática comtiana. ${ }^{12}$

\footnotetext{
${ }^{11}$ Alencar, "Alguns", 1898, pp. 113-114.

${ }^{12}$ Silva, "Análise", 1994, p. 32.
}

Las alianzas internacionales de Otto de Alencar no evitaron que sus ideas antipositivistas fueran rechazadas en la Escuela Politécnica de Río de Janeiro. La pronta reacción de los positivistas politécnicos, encabezados por Licínio Cardoso, al artículo de Alencar, dio inicio a una extendida polémica que se prolongó hasta la década de 1920. El impacto que causó el artículo de Otto de Alencar se debía no tanto a las correcciones que hacía a la matemática comtiana, como a la insinuación de que la enseñanza científica en la Escuela Politécnica de Río de Janeiro era anacrónica.

Además, en el plano epistemológico el artículo denunciaba que el positivismo comtiano, al proponer límites definitivos a la ciencia, propiciaba que intereses externos interfiriesen, indebidamente, en los problemas concernientes a la investigación científica. El propio Comte había sido incapaz de establecer una distinción coherente entre ciencia y opinión, faltando así al respeto a los principios fundamentales de la objetividad y la neutralidad científica. Aun así, por lo que a esto respecta, es preciso recordar que Comte nunca ocultó que subordinaba el desarrollo de la investigación científica a los imperativos de la reforma social positivista. Como nos enseña el historiador de la filosofía Émile Bréhier, para Comte

las ciencias positivistas son un medio para la reforma social; de manera directa o no, todas están ordenaclas en relación con ese fin, y si bien éste no impide que la certeza de las ciencias positivistas sea encontrada solamente a través de la observación y clel racio- 
cinio, lo que en nada afecta a la objetividad de los resultados, puede afectar, y de hecho en Comte afecta profundamente, la dirección y los límites de sus investigaciones. Existe, en todo el Cours de pbilosophie positive, un conflicto subyacente entre la libertad radical de la ciencia y las exigencias que se le imponen como medio; por más que, en virtud de tal conflicto, una doctrina que no parece haber sido ideada para introclucir el espíritu positivo en todos sus dominios, o ignora o critica ciertas direcciones efectivas de las ciencias, y restringe, a veces, el campo de la investigación. ${ }^{13}$

De esa manera, los politécnicos estaban obligados a escoger entre la "libertad radical de la ciencia" y las exigencias "restrictivas" de la doctrina positivista. Otto de Alencar y toda la generación de críticos del positivismo optaron por lo primero. Su distanciamiento del positivismo comtiano por "razones de orden matemático", llamaba la atención hacia un problema crucial que se les planteaba a los científicos brasileños de ese entonces. Necesitaban demostrar el valor intrínseco de la ciencia. Y que la investigación científica no solamente tenía valor como un medio para la "reforma social", sino que, esencialmente, lo poseía por su capacidad ilimitada de teorización y de resolución de problemas no necesariamente prácticos. La ciencia pura sería la ciencia liberada de los "compromisos sociológicos" propuestos por Comte.

${ }^{13}$ Bréhier, História, 1977, p. 257.

\section{CIENCIA PURA FRENTE A CIENCIA APLICADA}

Amoroso Costa, profesor catedrático de trigonometría, astronomía teórica y práctica geodésica en la Escuela Politécnica, tradujo para la nueva generación de politécnicos el mensajc que contenía la demostración de Otto de Alencar de los "errores de matemática" cometidos por Comte. Para él, la aceptación incondicional del positivismo comtiano significaba, paradójicamente, elevar la ciencia a la condición de manifestación superior del espíritu humano y, al mismo tiempo, condenarla a ser un orden estático confinado básicamente en el siglo XVIII. Esa "contradicción" condenaría al cicntífico brasileño a la esterilidad, pucsto que nada original podría producir, o porque, por otro lado, comprometería seriamente el proyecto de afirmación social del grupo. La "Conferencia sobre Otto de Alencar", ofrecida por Amoroso Costa el 29 de abril de 1918 en la Escuela Politécnica fue, en ese sentido, un verdadero manifiesto de la nueva generación de politécnicos. Repitiendo aquello que Otto de Alencar había dicho a través de ecuaciones matemáticas, afirmaba, entonces, que

Aceptar la Sintesis subjetiva equivale a rechazar toda la obra matemática del siglo pasado, la obra de Gauss y de Abel, de Cauchy y de Riemann, de Poincaré y de Cantor. Al tiempo que el primer tomo de la Filosofía positiva es un cuadro magistral de la ciencia matemática de fines del siglo XvII, la Síntesis, escrita cuando Comte ya estaba seducido por su construcción sociológica, es una de las tentativas más arbi- 


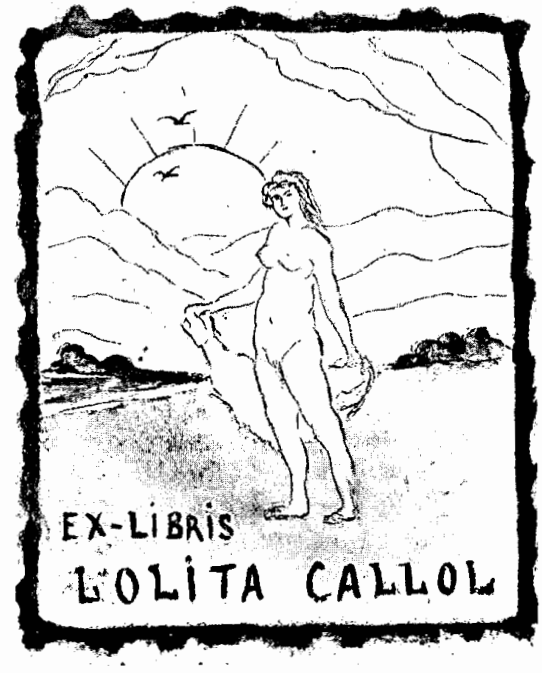

trarias que jamás se hayan hecho, de someter el pensamiento a fronteras artificiales $[\ldots]$ Forzoso es reconocer, como lo hace un insigne pensador, que nuestra aptitud para formularnos preguntas es superior a nuestros medios para resolverlas. A medida que el campo de la ciencia se dilata, surgen problemas cada vez más complejos, y la verdad absoluta escapa a nuestro entendimiento. ¿Cómo, sin embargo, concluir de ahí que el hombre se va a resignar a pensar dentro de los límites que fácilmente puede traspasar, aun en el caso mismo de que ello parezca justificable por motivos sociales o morales de cualquier ínclole? Nada se puede imaginar más seco y triste que una ciencia reclucicla a un estado de momia y a una clisciplina que niega al pensamiento un precioso derecho. ${ }^{14}$

${ }^{14}$ Costa, "Conferência", 1918, pp. 5-6.
El "precioso derecho" reivindicado por Amoroso Costa es el de la práctica de una ciencia que transcendiera los condicionamientos sociales por la fuerza de su lógica inmanente. Yendo en contra de la mentalidad pragmática, por lo que tocaba a la ciencia, enraizada entre los intelectuales brasileños desde finales del siglo xvin, y obviamente reforzada por el potitivismo, Amoroso Costa ensalzaba en Otto de Alencar precisamente la actitud opuesta:

Difícilmente hallaréis entre nosotros a alguien que se haya preocupado tanto por las cosas abstractas y clistantes de las aplicaciones, como ese hombre raro, cuya vida se centró en el pensamiento clesinteresado. ${ }^{\text {is }}$

Coherente con su trayectoria intelectual marcada por los estudios de lógica matemática, como sale a relucir en el ensayo "Las ideas fundamentales de la matemática" (1929) -un balance de las principales teorías matemáticas, inspirado en las nociones de deducción y raciocinio que defendía el matemático y filósofo de lá ciencia Henri Poincaré-, Amoroso Costa reivindicaba la creación de una "Facultad Superior de Ciencias" dedicada exclusivamente a la formación de científicos, sin compromiso ninguno con la formación técnica y profesional, idea que en ese mismo momento estaba siendo defendida por el ingeniero Luiz Cantanhede, profesor de la Escuela Politécnica que propugnaba el restablecimiento de los modestos cursos de ciencias matemáticas y físi-

${ }^{15}$ Ibid., p. 1. 
cas que existieron en la Escuela Politécnica de Río de Janeiro hasta 1896.

Aun habiendo conseguido sensibilizar a los positivistas sobre la necesidad de los cursos de ciencia pura, Amoroso Costa permanecía escéptico en cuanto a la viabilidad de tal proyecto. Alegaba que el medio cultural brasileño era todavía un terreno "impropio para el cultivo de esa suprema flor del espíritu que es la ciencia contemplativa y desinteresada". ${ }^{16}$ Esa opinión, paradójicamente, se apoyaba en una visión evolucionista típicamente positivista. Para él, en los países de civilización reciente, los rasgos que marcaban la cultura moderna -el utilitarismo y el pragmatismo- estaban condenados a ser transformados en fanatismo del progreso material:

En los países nuevos ese fanatismo es llevado a su apogeo, y esas mismas personas instruidas ignoran por completo que exista un ideal científico superior al del hombre que fabrica mil automóviles por día, o al de quien opera una apendicitis en diez minutos. De ahí la opinión que es casi unánime entre nosotros: la ciencia es útil, porque de ella precisan los ingenieros, los médicos, los industriales, los militares; pero no vale la pena hacerla en Brasil, porque es más cómodo y más barato importarla de Europa, en la cantidad que fuere estrictamente suficiente para nuestro consumo. Tal mentalidad es la que predomina entre aquellos que nos educan $\mathrm{y}$, por razones aún más vigorosas, entre aquellos que nos gobiernan. ${ }^{17}$

\footnotetext{
${ }^{16}$ Costa, "Ciência", 1923, p. 151.

${ }^{17}$ Ibid.
}

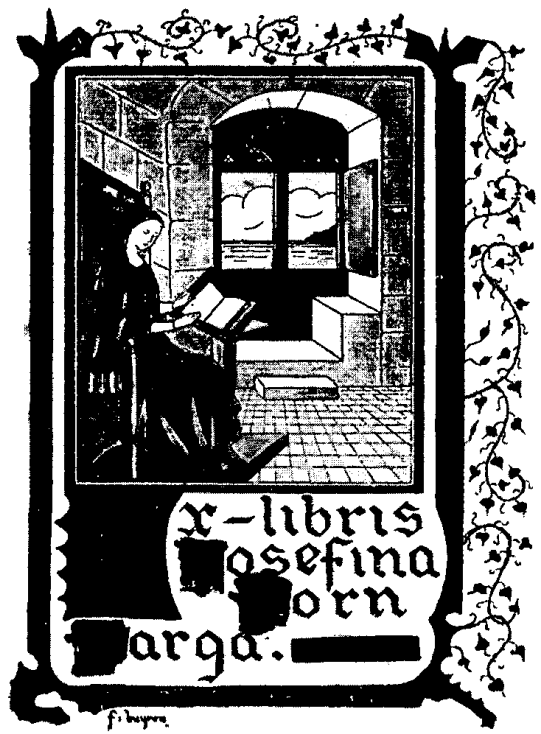

Por consiguiente, no iba a ser suficiente convencer a los positivistas de la validez de la ciencia pura, sino que era preciso ir más lejos y combatir la mentalidad ultrapragmática predominante entre los intelectuales y las clases dirigentes, que eran el principal obstáculo a vencer por aquellos que en ese momento luchaban en Brasil por el desarrollo de la ciencia desinteresada.

La repercusión de las ideas de Otto de Alencar y de Amoroso Acosta no quedó restringida a los medios politécnicos. El médico y fisiólogo Miguel Osório de Almeida, también uno de los fundadores de la Academia Brasileña de las Ciencias, estaba plenamente de acuerdo con las críticas enderezadas por los ilustres matemáticos contra el positivismo reinante en la cultura científica brasileña. En el artículo titulado 
"El ideal de los matemáticos", publicado en 1925, Miguel Osório evaluaba negativamente la influencia del positivismo en la enseñanza de las matemáticas en Brasil. Consideraba que la enseñanza de tal disciplina

se encontraba hasta hace poco, y tal vez aún se encuentre en gran parte, impregnada de las ideas positivistas. A la influencia de esas icleas escapan, cierto es, algunos jóvenes brillantes que representan la matemática en Brasil; mas, de manera general, dichas ideas muestran todavía una acción considerable en la dirección de nuestro pensamiento matemático. Y, sin embargo, la filosofía matemática cle Comte no es que sea deficiente hoy; ya lo era en el momento en que fue escrita. ${ }^{18}$

A pesar de que denunciaba el anacronismo científico del positivismo, Miguel Osório consideraba que la pugna entre los utilitaristas y los idealistas era un equívoco que perjudicaba el desarrollo de la ciencia brasileña. Menos radical que Amoroso Costa, él condenaba tanto los excesos de los bombres prácticos, que despreciaban ostensiblemente las preocupaciones desinteresadas de la ciencia pura, declarándolas inútiles y vanas, y tachando de soñadores inofensivos a quienes se dedicaban a ellas, ${ }^{19}$ como aquellos bombres de ciencia artistas, que desprecian por completo las aplicaciones prácticas de la ciencia, por considerarlas nocivas y perniciosas.$^{20}$ Miguel Osório defendía la idea de una recon-

\footnotetext{
${ }^{18}$ Almeida, "Ideal", 1925, pp. 81-82.

${ }^{19}$ Ibial., p. 129.

${ }^{20}$ Almeida, "Sciencia", 1925, p. 127.
}

ciliación entre los científicos prácticos y los científicos puros.

La postura conciliatoria de Miguel Osório parece ser la que mejor corresponde al estado de ánimo que predominaba en la Academia Brasileña de las Ciencias, que tuvo como primer presidente de la Sesión de Ciencias Matemáticas al notorio positivista Licínio Cardoso. El objetivo principal de aquellos científicos fue el dc transformar la opinión de los intelectuales y de las clases dirigentes respecto a la importancia de la investigación científica cuya utilización práctica no fucse inmediata ni directa.

\section{CONCLUSIÓN}

El hecho de que el positivismo haya sido el blanco predilecto de quienes militaban en favor de la ciencia putra, es un indicio de que ese sistcma científico-filosófico era un esquema intelectual profundamente enraizado entre los científicos brasileños de la época. Incluso aquellos que se declaraban antipositivistas, manifestaban un cicrto modo de pensar positivista. Sucesivas generaciones de politécnicos se hallaban comprometidas con cl positivismo asimilado durante el proceso de formación intelectual y profesional. De esa manera, la reconciliación entre positivistas y antipositivistas en el interior de la Academia Brasileña de las Ciencias reproduce, en menor escala, la dinámica de las pugnas dentro del campo científico, en torno a las ideas de Augusto Comte. Resulta significativo, por ejemplo, que el presidente de la Academia Brasilcña de 
las Ciencias, Henrique Morize, profesor catedrático de física experimental en la Escuela Politécnica y director del Observatorio Nacional, haya utilizado, en tres situaciones distintas, una misma cita de Augusto Comte para certificar el valor de la ciencia pura. El recurso al pensamiento comtiano como estrategia de persuasión demuestra que el positivismo servía también a los intereses de los antipositivistas.

Los positivistas, tradicionales detentadores del poder institucional en la Escuela Politécnica de Río de Janeiro, no podían ser simplemente despreciados por los militantes de la causa de la ciencia pura. La demostración del anacronismo científico de Augusto Comte, que se empleó como estrategia de afirmación del científico puro, no sacudió el prestigio intelectual de los positivistas, que acabaron por ocupar sitios importantes en la Academia Brasileña de las Ciencias. Así, el positivismo fue el eslabón de enlace entre dos generaciones de científicos: una formada por politécnicos dedicados a las actividades del campo de la ingeniería, que valorizaba la ciencia aplicada como un instrumento dirigido a la modernización de la sociedad; y otra constituida por jóvenes politécnicos dedicados a los estudios matemáticos, que se empeñó en la defensa de la ciencia pura como una estrategia que apuntaba a su afirmación en el campo científico.

\section{BIBLIOGRAFIA}

Alencar, Otto de, "Alguns errors de mathemática na Syntesi Subjectiva de Augusto
Comte", Revista de la Escuela Politécnica, vol. 9 , núm. 10, 1898, pp. 113-130.

Almeida, Miguel Osório de, "O ideal dos mathematicos" en Homens e coisas da sciencia, Cia Graphica/Editora Monteiro Lobato, São Paulo, 1925.

" $\Lambda$ sciencia pela sciencia" en Homens e coisas da sciencia, Cia. Graphica/ Editora Monteiro Lobato, Sĩo Paulo, 1925.

Bourdieu, Pierre, A economia das trocas simbólicas, Editoral Perspectiva, São Paulo, 1987.

Bréhier, Émile, "El siglo XIX: periodo de los sistemas, 1800-1850", História da Filosofia, Mestre Jou, São Paulo, 1977, t. 2, fasc. 3 .

Cardoso, Licínio Atanásio, Evolução da mathemática no Brazil, Typ. G. Leuzinger, Río de Janeiro, 1887.

Costa, Amoroso, "Conferencia sobre Otto de Alencar", Revista Didática da Escola Polytécnica, núm. 13, 1918, pp. 3-24.

, "Pela ciência pura". Idéias fundamentais da matemática e outros ensaios, Eclitora Convívio \& Edusp., São Paulo, 1923.

Dantes, Maria Amélia, "Fases de la implantación de la ciencia en Brasil", QuipuRevista Latinoamericana de las Ciencias y la Tecnología, vol. 5, núm. 2, 1988, pp. 265-276, México.

Ferreira, Luiz Otávio, "Os politécnicos: ciência e reorganização social segundo o pensamento positivista da Escola Politécnica do Rio de Janeiro, 1862-1922", tesis de maestría, IFCS-UFJR, 1989.

Lins, Ivan, História do positivismo no Brasil, Cia. Editora Nacional, São Paulo, 1967.

Paulinyi, Eno, Esboço bistórico da Academia Brasileira de Ciências, Consejo Nacional de Investigación-CNPq, Brasilia, 1989.

Silva, Clóvis Pereira da, " $\Lambda$ nálises de dois textos de Otto de Alencar", Revista da Sociedade Brasileira de História da Ciência, núm. 12, 1994, pp. 27-38. 


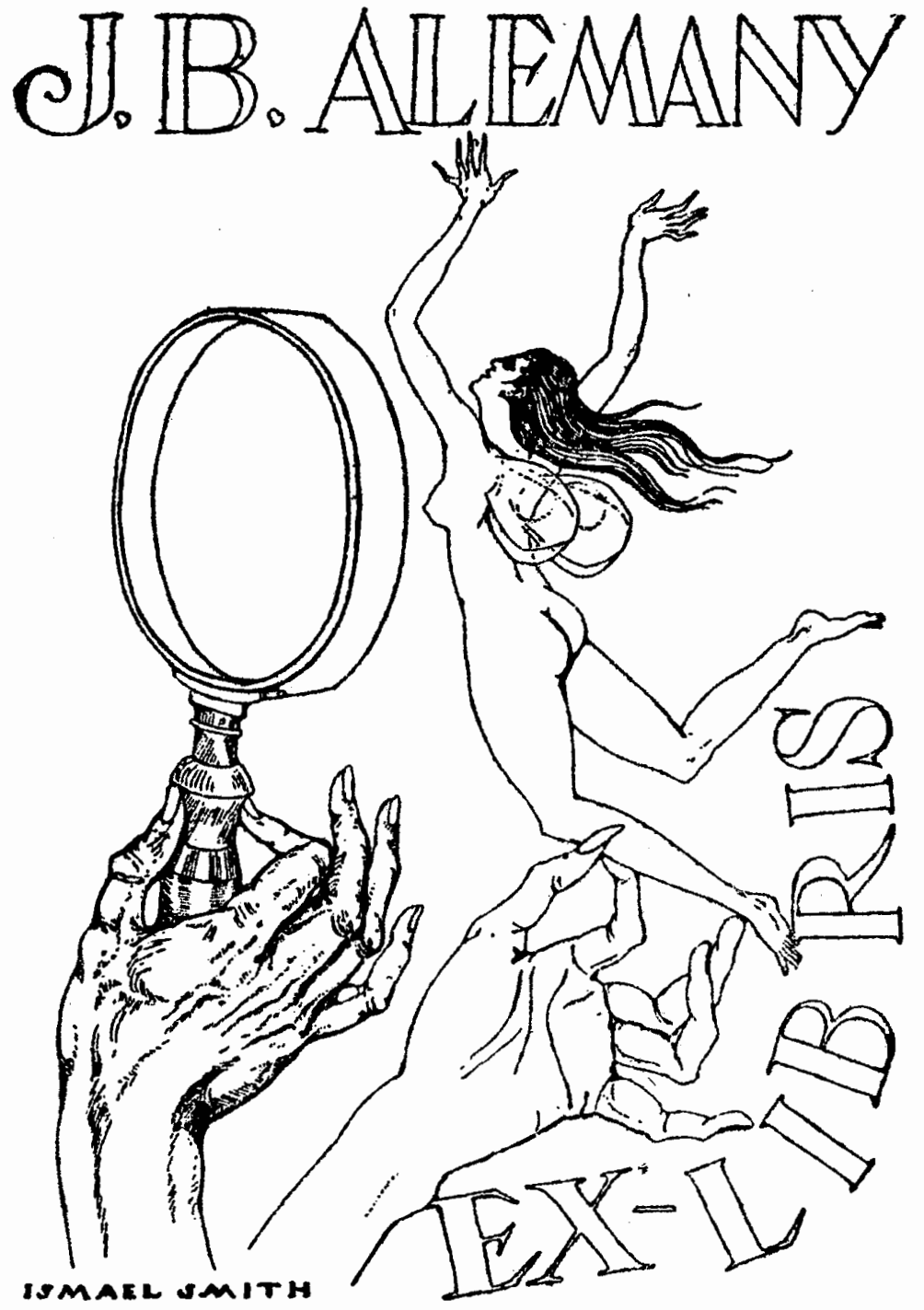

